Revue d'histoire de l'Amérique française

FB. REVUE D.HISTOIRE DE L'AMÉRIQUE FRANÇAISE

\title{
Biographie, narration et philosophie de l'histoire
}

\section{Julien Goyette}

Volume 54, numéro 1, été 2000

URI : https://id.erudit.org/iderudit/305657ar

DOI : https://doi.org/10.7202/305657ar

Aller au sommaire du numéro

\section{Éditeur(s)}

Institut d'histoire de l'Amérique française

\section{ISSN}

0035-2357 (imprimé)

1492-1383 (numérique)

Découvrir la revue

\section{Citer ce document}

Goyette, J. (2000). Biographie, narration et philosophie de l'histoire. Revue

d'histoire de l'Amérique française, 54(1), 81-88. https://doi.org/10.7202/305657ar d'utilisation que vous pouvez consulter en ligne.

https://apropos.erudit.org/fr/usagers/politique-dutilisation/ 


\title{
Biographie, narration et philosophie de l'histoire
}

JULIEN GOYETTE

Département d'histoire

Université du Québec à Montréal

\begin{abstract}
T es historiens sont d'ordinaire inconfortables avec le genre biogra$\checkmark$ phique, celui-ci restant pour eux quelque peu inavouable. Il faut dire que la biographie historique se présente couramment comme un problème non résolu, peut-être même une cause perdue. Et il n'y a rien là d'étonnant: à répétition, en ce siècle, des penseurs ont mis en doute la valeur de ses explications; ils lui ont reproché de faire l'histoire des grands hommes au détriment de l'histoire des hommes et, plus récemment, des femmes; ils ont dénoncé - et souvent avec raison - sa langueur, sa prolixité, ses faux-semblants, etc. Bref, si elle a été longtemps présentée comme le modèle même des écrits historiques, la biographie se montre plutôt chancelante au $\mathrm{xx}^{\mathrm{e}}$ siècle. Aujourd'hui, à un moment où rien pourtant ne nous permet de douter de la solidité de son lectorat, le genre paraît encore instable, n'arrivant à faire l'unanimité ni chez les scientifiques ni chez les littéraires ${ }^{1}$.

Tout compte fait, c'est probablement Claude Lévi-Strauss qui a le mieux transcrit ce que d'aucuns considèrent être la tare congénitale de la

1. "Les biographes sont de la racaille!», dit Milan Kundera. Et il ajoute: "[...] fouiller dans la correspondance intime, interroger les anciennes maitresses, convaincre des médecins de trahir le secret médical, c'est dégueulasse. » Milan Kundera, cité par Colette Cosnier, «Les pièges de la biographie ", dans Marta Dvorak, dir., La création biographique/Biographical Creation (Rennes, Presses universitaires de Rennes et Association française d'études canadiennes, 1997), 25.
\end{abstract}


biographie historique: "L'histoire biographique et anecdotique est la moins explicative; mais elle est la plus riche du point de vue de l'information, puisqu'elle considère les individus dans leur particularité [...] le choix relatif de l'historien n'est jamais qu'entre une histoire qui apprend plus et explique moins, et une histoire qui explique plus et apprend moins ${ }^{2}$.» Face à un tel dilemme, on comprend que plusieurs se soient laissés convaincre qu' «il y a la biographie, qui traite l'homme individuellement, et l'histoire, qui le traite comme partie d'un ensemble, et que la bonne biographie fait la mauvaise histoire ${ }^{3}$.»

Pour nombre de raisons, cette antinomie entre l'histoire informative (dite aussi événementielle, anecdotique ou narrative) et l'histoire explicative (sociale, structuraliste) n'a pas été totalement surmontée. En regard du sens commun, la biographie demeure un genre historique mineur, et la connaissance biographique reste dévaluée, entre autres parce qu'assimilable à la "stérile» narration. Pourtant, des acquis de l'épistémologie de l'histoire ont considérablement bousculé les habituelles conceptions de la narration. De même, l'évolution de l'historiographie a prouvé la capacité de la biographie à perdurer, même dans des conditions qui lui sont défavorables. C'est ce qui me fait croire qu'il est possible de redonner une certaine respectabilité épistémologique à la biographie, et que cette dernière n'est pas qu'un simple problème de l'historiographie à résoudre, mais bien un aspect incontournable de l'histoire et de la pratique historique.

Depuis quarante ou cinquante ans, les thèses narrativistes se sont largement répandues en épistémologie de l'histoire. En cherchant à s'opposer aux tenants des modèles d'explication de type déductifnomologique - qui tentaient de réconcilier les sciences dites «dures» et "humaines" sous la bannière des systèmes de lois universelles - des philosophes ont réhabilité la narration, une forme de discours touchée de discrédit depuis Descartes ${ }^{4}$ et associée abusivement à l'histoire traditionnelle par plusieurs historiens du $\mathrm{xx}^{\mathrm{e}}$ siècle. Pour dire vite, les thèses narrativistes procèdent d'un constat apparemment banal: c'est-à-dire

2. "L'histoire biographique [...] est une histoire faible, qui ne contient pas en elle-même sa propre intelligibilité, laquelle lui vient seulement quand on la transporte en bloc au sein d'une histoire plus forte qu'elle [...]». Claude Lévi-Strauss, La pensée sauvage (Paris, Plon, 1962), 346347.

3. Edward H. Carr, Qu'est-ce que l'histoire? (Paris, Éditions La découverte, 1988), 98.

4. Maurice Lagueux, "Narrativisme et philosophie spéculative de l'histoire", Revue de synthese, 4,1 (janvier-mars 1998): 65 . 
que la narration est en soi une forme d'explication, et que par voie de conséquence, raconter correctement, c'est déjà expliquer. Cette observation, que l'on doit avant tout au philosophe analytique Arthur Danto ${ }^{5}$, implique que la narration, loin d'être un simple artifice stylistique imputable à l'historien, ait un rôle déterminant à jouer dans le procédé explicatif en histoire. Le récit historique ainsi appréhendé, l'opposition entre la narration et l'explication s'estompe. En fait, plutôt que de disparaître, elle se déplace entre la narration et la chronique, cette dernière étant vue comme la stricte exposition des faits sans aucun lien explicatif.

Du point de vue historique, cette distinction entre l'activité d' «établir les faits » et celle de les «mettre en œuvre » n'a rien de très original. Déjà en 1897, Henri Pirenne faisait la différence entre l'histoire-érudition et l'histoire-récit ${ }^{6}$. Du reste, ce qui est novateur dans les théories narrativistes, c'est d'attribuer a priori les virtualités explicatives au récit, plutôt que de les réserver aux seules relations causales.

Mais qu'est-ce qu'un récit et, surtout, qu'a-t-il à voir au juste avec la biographie? Par convention, si j'ose dire, on puise de la Poétique d'Aristote la définition du récit. Pour le philosophe, l'histoire représente "l'agencement des actes accomplis ${ }^{7}$ ", en autant que ces mêmes actes forment un tout, une totalité. Or, ajoute-t-il, "forme un tout, ce qui a commencement, milieu et fin $^{8} »$. Du coup, les choses se précisent à propos du récit: il s'agit d'une relation close d'actes accomplis. Mais il manque encore un élément fondamental. Il consiste en ce que les auteurs appellent généralement l'intrigue (plot en anglais), c'est-à-dire ce qui lie les différents temps du récit et rend possible le dénouement de l'histoire racontée?.

5. Arthur Danto, Analytical Philosophy of History (New York, Cambridge University Press, 1968), 201.

6. "L'authenticité des textes établie, les sources critiquées, la chronologie des événements fixée, il reste encore à faire l'histoire." Henri Pirenne, "Une polémique historique en Allemagne", Revue historique, 64 (mai-août 1897): 50.

7. Aristote, cité dans Maurice Lagueux, loc. cit., 69.

8. Ibid., 70 .

9. En liant récit et histoire, on rendait évidemment inévitable l'association avec la fiction; elle n'a pas échappé à certains auteurs, particulièrement pressés, il faut dire, de saper la première prétention de l'historien: celle de raconter fidèlement ce qui s'est passé. Voir là-dessus, entre autres, les travaux controversés d'Hayden White. L'historien, quant à lui, n'est pas tenu de tirer des conséquences aussi radicales des énoncés narrativistes, même si, comme le reconnaît Roger Chartier, le "chemin est [...] forcément étroit pour qui entend refuser, en même temps, la réduction de l'histoire à une activité littéraire de simple curiosité, libre et aléatoire, et la définition de sa scientificité à partir du seul modèle de la connaissance du monde physique». Roger Chartier, Au bord de la falaise. L'histoire entre certitudes et inquiétude (Paris, Albin Michel, 1998), 104. 
À première vue, ne peut-on pas dire que l'objet de la biographie s'accorde admirablement avec cette notion de récit? Ne peut-on pas prétendre que la vie d'un humain s'organise, et de façon obligée, en fonction d'un commencement (la naissance), d'un milieu (le cours de la vie) et d'une fin (la mort)? Les péripéties qui ponctuent nos vies, comme celles des agents historiques, ne peuvent-elles pas être entendues telles de vastes intrigues aux multiples rebondissements?

Que la notion de récit paraisse autant à la mesure de l'existence humaine ne doit pas cependant nous méprendre sur la nature précise de l'un ou de l'autre. La corrélation narration-explication peut être facilement admise - du moins pour l'auteur de ces lignes. Mais peut-on pour autant considérer la réalité (l'histoire vécue) comme étant elle-même structurée à la façon d'un récit ${ }^{10}$ ? Ou, pour rester plus près des préoccupations historiennes, est-il possible de voir dans la vie d'un individu un cadre d'analyse opératoire? Recevoir une catégorie comme celle de vie, sans la questionner, c'est évidemment passer à côté de plusieurs implications cruciales. Des implications qui n'ont pas échappé à Pierre Bourdieu :

Parler d'histoire de vie, c'est présupposer au moins, et ce n'est pas rien, que la vie est une histoire et que, comme dans le titre de Maupassant, Une vie, une vie est inséparablement l'ensemble des événements d'une existence individuelle conçue comme une histoire et le récit de cette histoire. C'est bien ce que dit le sens commun, c'est-à-dire le langage ordinaire, qui décrit la vie comme un chemin, une route, une carrière, avec ses carrefours (Hercule entre le vice et la vertu), ses embûches, voire ses embuscades (Jules Romains parle des "embuscades successives des concours et des examens"), ou comme un cheminement, c'est-à-dire un chemin que l'on fait et qui est à faire, un trajet, une course, un cursus, un passage, un voyage, un parcours orienté, un déplacement linéaire, unidirectionnel (la «mobilité »), comportant un commencement ( "un début dans la vie»), des étapes, et une fin, au double sens, de terme et de but («il fera son chemin" signifie qu'il réussira, il fera une belle carrière), une fin de l'histoire. C'est accepter tacitement la philosophie de l'histoire au sens de succession d'événements

10. C'est l'hypothèse du philosophe David Carr, pour qui la " configuration du temps par le récit et la constitution de soi en tant que sujet narratif ne sont plus des choses qui se font dans ou par les communautés, c'est plutôt ce qu'elles sont, c'est leur manière d'être. Donc, en tant que concept ontologique, le récit est une manière de caractériser, non pas la connaissance historique, mais l'objet de cette connaissance, la réalité historique, les res gestae elles-mêmes ". Voir David Carr, "Épistémologie et ontologie du récit", dans Jean Greisch et Richard Kearney, dir., Paul Ricoeur: les métamorphoses de la raison herméneutique (Paris, Éditions du Cerf, 1991), 212. 
historiques, Geschichte, qui est impliquée dans une philosophie de l'histoire au sens de récit historique, Historie, bref, dans une théorie du récit, récit d'historien ou de romancier, sous ce rapport indiscernables, biographie ou autobiographie notamment ${ }^{11}$.

Aussi déplaisantes qu'elles puissent paraître aux yeux des historiens, les philosophies de l'histoire n'en répondent pas moins à une exigence que l'histoire faillit elle-même à satisfaire tout à fait: clore les narrations et donc, si l'on souhaite rester cohérent avec la théorie narrativiste, les explications. Et cela parce que l'historien, quelle que soit sa spécialisation, se bute inévitablement à ce qui semble être un paradoxe de l'histoire. Maurice Lagueux le caractérise ainsi : "[...] exprimer de manière exacte - et en ce sens définitive - l'état d'inachèvement d'un monde inachevé, du fait qu'il demeure toujours susceptible de se révéler autre que ce que, en cet état d'inachèvement, il laisse penser qu'il sera ${ }^{12}$. " Malgré la formulation typiquement philosophique de l'énoncé, on ne perçoit que trop facilement l'ampleur du problème. Dans le cas précis de la biographie, il se pose en ces termes: comment donner un caractère fini au récit d'une existence mortelle que l'histoire déborde en amont et en aval et qui, au surplus, reste susceptible de changer de sens en regard d'événements présents ou futurs? Qui, par exemple, oserait prétendre que la vie de Lénine a exactement le même sens depuis l'écrasement du régime soviétique? Comment passer à côté du fait que Lénine ait, en quelque sorte, rendu possible Staline ${ }^{13}$ ?

Transposé sur le plan de l'histoire sociale, ce problème prend un autre tour. On peut se demander, en effet, comment faire cohabiter le temps long et le temps court sans discriminer l'un ou l'autre au moment de conclure la narration. Cette dernière difficulté trouve un exemple fameux dans La Méditerranée et le Monde méditerranéen à l'époque de Philippe II. Il nous est révélé par Paul Ricoeur dans le premier tome de Temps et récit. Préoccupé surtout par la longue durée, Fernand Braudel n'en termine pas moins son monumental ouvrage par la mort de Philippe II. Or, de l'aveu même de l'auteur ${ }^{14}$, et du "point de vue de la

11. Pierre Bourdieu, "L'illusion biographique", Actes de la recherche en sciences sociales, 62-63 (juin 1986): 69 .

12. Maurice Lagueux, loc. cit., 85 .

13. Paul Ricoeur, "Table ronde/Round table. Temps et récit, volume I", Revue de l'Université d'Ottawa/University of Ottawa Quarterly, 55,4 (1985): 317. Voir aussi Maurice Lagueux, loc. cit., 74-75.

14. "Je ne crois pas que le mot de Méditerranée ait jamais flotté dans son esprit [celui de Philippe II] avec le contenu que nous lui suggérons. Une véritable géographie ne faisait pas 
grande histoire de la Méditerranée, cette mort n'est pas un grand événement ${ }^{15}$ ». Le personnage central du récit de Braudel n'est-il pas un espace maritime, la Méditerranée pour être plus précis? En ce qui concerne les contemporains du roi Philippe II, pourtant, il est indéniable que cette mort fut un événement déterminant. On le voit: incompatible avec une explication s'inscrivant dans le temps "de la grande histoire", telle que la concevait Braudel, le destin individuel n'arrive pas à s'effacer complètement du champ de prospection de l'historien. Et Ricœur de conclure: "Et sans la mort qui tranche un tel destin, saurions-nous encore que l'histoire est celle des hommes ${ }^{16}$ ?"

Les philosophies de l'histoire, telles qu'évoquées plus haut par Bourdieu, ont justement la prétention de résoudre ces paradoxes de l'histoire en plaquant sur celle-ci des trames qu'elles souhaiteraient nous rendre vitales et irrésistibles. Qu'il s'agisse des théories hégéliennes, des idées de Marx, de Spengler ou de Toynbee, des dogmes du libéralisme, toutes ces philosophies visent à donner un sens prescrit à l'histoire humaine, à clore définitivement la connaissance que nous avons de nous-mêmes. N'est-ce pas d'ailleurs ce qui autorise Francis Fukuyama à proclamer sans pudeur la fin de $l^{\prime}$ histoire ${ }^{17}$ ?

Que les philosophies de l'histoire répugnent aux historiens, c'est évidemment compréhensible; qu'ils arrivent complètement à s'en passer, c'est cependant moins sûr. Et cela parce qu'à un moment ou à un autre, tous les examinateurs du passé doivent se référer à ces sortes d' " arrière-plans" pour situer leurs reconstitutions et contrer les effets de la contingence. Ainsi, le biographe est-il contraint de clore ses narrations, de donner simultanément à son objet d'étude une intelligence en regard du passé, du présent et même, du futur. En regard de tout cela, par contre, il apparaît que la biographie n'a plus guère à envier du point du vue épistémologique aux autres grands genres historiques. Son caractère éminemment narratif, comme sa dépendance envers les philosophies de l'histoire, en constituent la preuve et le gage. Parce que s'il reste

partie de l'éducation des princes. Toutes raisons suffisantes pour que cette longue agonie, terminée en septembre [15]98, ne soit pas un grand événement de l'histoire méditerranéenne... Pour que se marquent à nouveau les distances de l'histoire biographique à l'histoire des structures et encore plus à celles des espaces." Fernand Braudel, cité en note dans Paul Ricoeur, Temps et récit, 1: L'intrigue et le récit historique (Paris, Seuil, 1983), 376.

15. Ibid.

16. Ibid.

17. Francis Fukuyama, La fin de l'histoire et le dernier homme (Paris, Flammarion, 1992), $451 \mathrm{p}$. 
possible, en dernière analyse, de discuter du poids des individualités dans l'histoire, ou même de privilégier à bon droit les acteurs sociaux, il devient plus difficile toutefois de dénier toute faculté explicative à la biographie - en autant bien sûr qu'elle satisfasse en même temps les critères de scientificité de la discipline.

Cette reconnaissance du caractère explicatif du savoir biographique autorise, entre autres, à une réévaluation de l'apport de la biographie à l'histoire. Et tout porte à croire que cet apport est significatif: elle rappelle sans cesse la dépendance de l'histoire envers les conjonctures; elle permet d'éviter les travers d'un formalisme à outrance; elle aide à se prémunir d'une histoire qui serait désincarnée. Sinon, comment expliquer qu'autant d'historiens émérites, le plus souvent champions de l'histoire sociale, se soient laissés séduire par elle? En "partant de la "mauvaise" direction ${ }^{18}$ ", pour reprendre l'expression qu'utilise Ian Kershaw pour parler de lui-même, les Lucien Febvre ${ }^{19}$, Emmanuel Leroy-Ladurie, Jacques Le Goff ont tous abouti à la biographie, et cela ne s'est certainement pas fait sans qu'ils modifient leur conception et leur traitement de l'histoire. C'est d'ailleurs en méditant son Saint Louis, alors qu'il était confronté à la fois à la biographie hagiographique du xiII ${ }^{\mathrm{e}}$ siècle et à la biographie historique de la fin du $\mathrm{xx}^{\mathrm{e}}$ siècle, que Le Goff a pu vérifier la fausse opposition entre l'histoire narrative et l'histoire structuraliste $^{20}$. Au total, la remarque qui s'impose paraît être celle-ci: si toute démarche historique rigoureuse, telles celles, à n'en pas douter, de Febvre, de Leroy-Ladurie, de Le Goff ou de Kershaw, rencontre un jour la question de la biographie, c'est peut-être parce que cette question est partie prenante de l'histoire et que, sans la trace de l'individuel, l'histoire n'est plus vraiment l'histoire.

18. Ian Kershaw, Hitler 1889-1936: Hubris (Paris, Flammarion, 1999), 8.

19. Les biographies de Lucien Febvre annonçaient toutefois de sombres jours pour la biographie : "À une histoire intellectuelle des intelligences sans brides et des idées sans support, est opposée une histoire des représentations collectives, des outillages et des catégories intellectuels disponibles et partagés à une époque donnée [...] La biographie intellectuelle à la manière de Febvre est donc, en fait, histoire de la société puisqu'elle situe ses héros à la fois comme témoins et produits des conditionnements collectifs qui bornent la libre invention individuelle. La voie était ainsi ouverte (une fois abandonnée le goût particulier de Febvre pour la biographie) à une histoire des systèmes de croyances, de valeurs et de représentations propres à une époque ou un groupe, désignée dans l'historiographie française par l'expression d'autant plus englobante que son contenu notionnel reste flou, d'histoire des mentalités'." Roger Chartier, "Histoire intellectuelle et histoire des mentalités. Trajectoires et questions", Revue de synthèse: 3,111-112 (juillet-décembre 1983): 286.

20. Jacques Le Goff, Saint Louis (Paris, Gallimard, 1996), 17-18. 
Cela dit, sans doute la biographie n'occupera jamais qu'une part limitée de la production historique; il suffisait ici de rappeler que cette part est valable, essentielle et irréductible. Car si l'individuel, au même titre que l'événementiel, crée dans nos travaux plus que sa part d'" effets de réalité », ou d'“illusions" si l'on préfère, il n’en représente pas moins l'un des traits constitutifs de l'histoire auquel il est certainement vain d'essayer de passer outre. 